\title{
Analysis of Enterprise Sustainability and Control of Efficiency of Cattle-Breeding Enterprise Functioning
}

\author{
B.N. Shchetkin \\ Perm Institute of Economics and Finances, Perm, Russia, Ekaterininskaya Str., 141, Perm, Russia, 614068 \\ Email: smart051@rambler.ru
}

\section{Doi:10.5901/mjss.2015.v6n3s6p101}

\section{Abstract}

Under conditions of development of market relations, when resources' provision of each market subject depends on the efficiency of economy, the increase of economic effectiveness becomes a primary task. The main parameters of functioning efficiency in modern conditions are stable state of enterprises in the markets and adapting the systems of production and management in a dynamic external environment. A basis of the system of internal control of enterprise functioning efficiency is technical, ecological, and economic information about its activity. The control procedure is conducted on the basis of the author's algorithm of evaluation of technical and eco-economic parameters which allow determining their efficiency during realization of ecological and economic programs of cattle-breeding enterprises; determining the order and sequence of operations in planned situation, and in case of deviations from permissible values - informing about the control results for timely decision making which removes these deviations.

Keywords: stable state; control; system of technical and eco-economic parameters.

\section{Introduction}

Technical and eco-economic system (TEES) possesses a structural dynamic and statistical sustainability, if the transformation of its structure doesn't lead to violation of technological, ecological, economic, and financial sustainability, while preserving the parameters of system functioning in the set limits of confidence band (allowed limits).

In order to provide the necessary and sufficient conditions for structural dynamic and statistical sustainability, they should be viewed in a certain sequence from the positions of systemic approach, i.e., in view of the goals of TEES [3].

At that, analysis of the enterprise sustainability and revealing the violations of balanced state in the process of monitoring lead to the necessity for controlling the efficiency of the cattle-breeding enterprise functioning (Fig. 1).

\section{Methods and Materials}

Let us present the parameter of sustainable development of cattle-breeding sector as a function of three variables:

$$
W=f\left(Y^{T}, Y^{\jmath}, Y^{\ni}\right)
$$

where:

$Y^{T}$ - variable, evaluating the technological sustainability of the sector;

$Y^{\hat{\jmath}}$ - variable, evaluating the ecological sustainability of the sector;

$Y^{\ni}$ - variable, evaluating the economic sustainability of the sector.

In order to decrease the degree of source data (data reduction), a factor analysis method is used. The sense of the factor analysis is to present the initial parameters $X$ in the form of certain sum of latent variables $F$ which are called factors: $X_{1}, X_{2}, \ldots, X_{m} \rightarrow F_{1}, F_{2}, \ldots, F_{p} \quad p<<m$, while optimal space of new orthogonal (mutually uncorrelated) variables is formed, without substantial loss of content information that is a part of source data. 


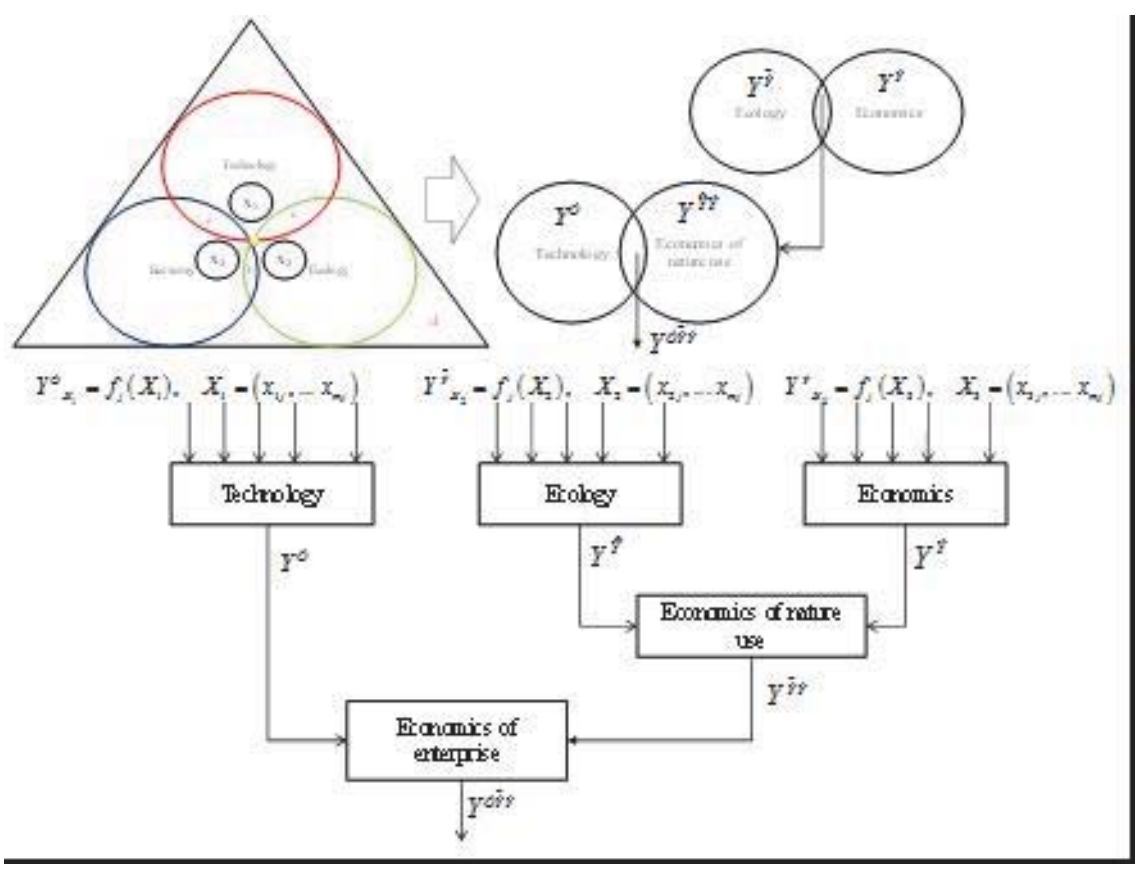

Fig. 1. Parameters of sustainable development of cattle-breeding sector

Extension of local integrated parameters of the state of the sector is conducted on the basis of geometric middling:

$W=f\left(Y^{T}, Y^{\hat{\jmath}}, Y^{\ni}\right)=\sqrt[3]{Y^{T}, Y^{\hat{\jmath}}, Y^{\ni}}$

where:

$Y^{T}$ - standardized variable, evaluating the technological sustainability of the sector;

$Y^{\hat{\jmath}}$ - standardized variable, evaluating the ecological sustainability of the sector;

$Y^{3}$ - standardized variable, evaluating the economic sustainability of the sector.

Extended integrated parameter of dynamic and structural sustainability of cattle breeding $W$ allows real-time tracking of the condition of sector's functioning and performing preventive actions for avoidance of sustainability loss. In order to evaluate how close the condition of technological, ecological, and economic spheres is to non-sustainable, it is necessary to consider the rates of changing $\bar{Y}_{t}^{T}, \bar{Y}_{t}^{\hat{\jmath}}, \bar{Y}_{t}{ }^{3}$, using the formula:

$\bar{Y}_{t}^{i}=Y_{\min }^{i} / Y_{t}^{i}$

where:

i - denotes technological, ecological, and economic sustainability;

$Y^{\prime}{ }_{\min }$ - specific, above the critical point, corresponding local parameters of sustainability.

Obviously, values ${ }^{\bar{Y}_{t}^{i}}$ vary from 0 to 1, besides $\bar{Y}_{t=1}^{i}$ corresponds to match of current value with critical value. While choosing a maximum value from $\bar{Y}_{t}^{T}, \bar{Y}_{t}^{3}, \bar{Y}_{t}^{\ni}$ during monitoring, we determine the sphere which is the most influenced by destructive actions. Monitoring also supposes comparison of the parameter values for the period $\Delta \mathrm{t}$.

\section{Discussion}

However, a large quantity of components of statistics $k_{y}$ for each realization $y(t)$ of initial parameter $Y^{\text {rjo }}(t)$ of TEES complicates the operating control of quality of the enterprise functioning. The control algorithms, built for full variety of values ${ }^{k_{y}}$ of enterprise functioning efficiency, can be practically realized during scientific research and functioning of TEES enterprise with the computer processing of the information. This requires determining the most appropriate evaluations of parameters of TEES functioning efficiency. This refers to the choosing from all variety of components $k_{y}$ such evaluations which would provide a simple and at the same time effective and relatively accurate operating control of 
technological and ecological processes in the formation of sustainable functioning of enterprise. It is also important to determine unified principles and parameters of control which would allow controlling the TEES quality. At last, the most appropriate evaluations open wide possibilities for automation of the processes of cattle-breeding management, using the control algorithm (Fig. 2).

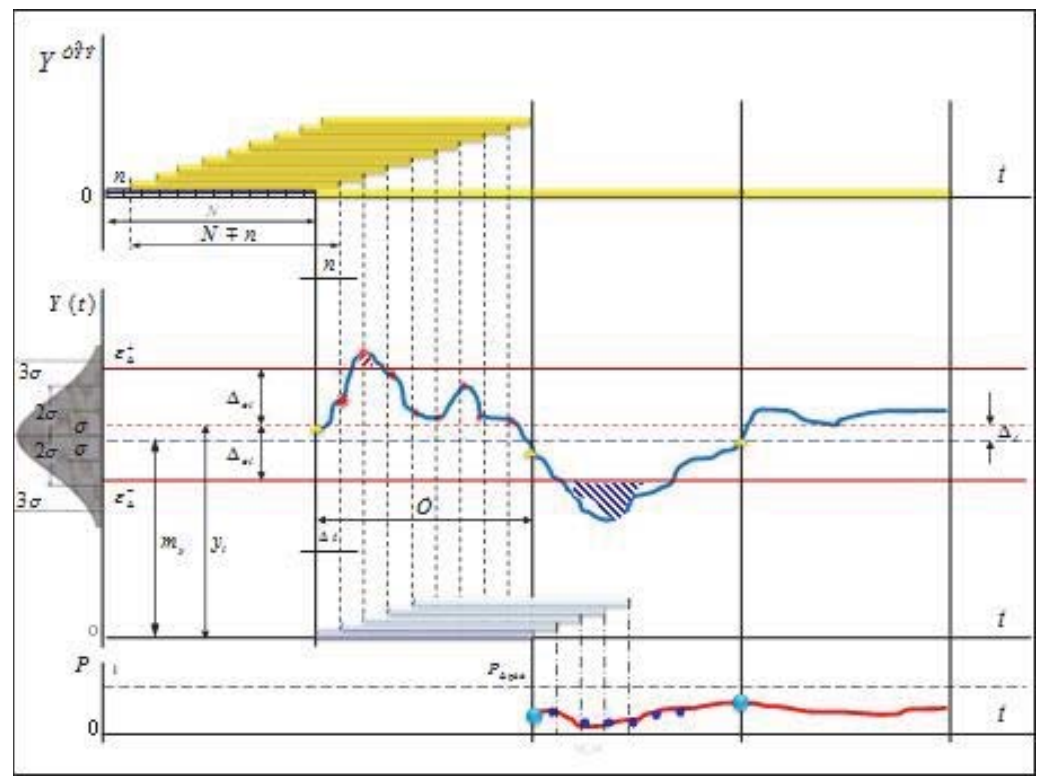

Fig. 2. Scheme of the control algorithm of dynamic and statistic sustainability of TEES

Using the moving average methods for evening out the time series, it is possible to eliminate the random fluctuations and find values which correspond to the influence of the main actors. Evening out with the help of moving average is based on the fact that averages liquidate the random fluctuations. This happens due to the substitution of primary levels of time series by arithmetic mean value within the chosen time period. The received value belongs to the middle of chosen period. Then the period shift by $n$ observation for set time step $\Delta t$, and calculation of the average continues - moreover, periods of determining the average are always equal $(N)$. Thus, the average is centered in every case, i.e. belongs to mid-point of evening-out interval and constitutes a level for this point.

During evening-out of time series by moving averages, all levels of the series are involved in the calculations. The wider the evening-out interval, the smoother the trend. At small values of $n$, variability of smoothed series increases. At the same time, the quantity of observations grows, which creates an opportunity for using data for calculation of dynamic and statistic sustainability of TEES.

The choice of evening-out interval depends on the goals of the research. At that, the most important factor is time period of the action and, consequently, the elimination of random factors' influence.

\section{Results}

Let us assume that the parameter which characterizes the efficiency of technological and ecological process functioning can be represented by realization of $y(t)$ - random function of $Y(t)$. Let us also assume that this function is stationary and ergodic. Then, the technical requirements or technical task suppose symmetrical absolute operational margins ${ }^{\Delta_{y}}$ for function deviation and its every realization $y(t)$ from the average value ${ }^{m_{y}}$, i.e.

$m_{y}-\Delta_{y} \leq y(t) \leq m_{y}+\Delta_{y}$.

Connection between permitted $\Delta_{y}$ and probability for its maintenance in the interval (4) is found from the expression

$$
P_{\Delta}=P\left[\left(m_{y}-\Delta_{l y}\right) \leq y(t) \leq\left(m_{y}+\Delta_{2 y}\right)\right]=\int_{m_{y}-\Delta_{y y}}^{m_{y}+\Delta_{y y}} f(y) d y
$$


under condition $\Delta_{l y}=\Delta_{2 y}=\Delta_{y}$

$$
P_{\Delta}=\int_{m_{y}-\Delta_{y}, m_{y}+\Delta_{y}}^{m_{y}} f(y) d y \text {. (6) }
$$

Probability of margin overshoot will constitute $\varepsilon_{\Delta}=1-P_{\Delta}$.

For operating control (with measuring system available) of the efficiency of cattle-breeding enterprise functioning, which is determined by the realization of $y(t)$ in the specific period $T$ of control, it is necessary, according to ( $y(t)=m_{y}+\dot{y}(t)$, to constantly receive information regarding realization of $y(t)$ and, during time interval $T$, define numerical characteristics of this realization and compare them with permitted at the set margin ${ }^{\Delta_{y}}$ and probability $P_{\Delta 3}$ $\left(\right.$ or $\left.\varepsilon_{\Delta C}\right)[2]$.

Algorithm of controlling the quality of technological process of cattle-breeding production is related to adjusting value $y_{H}$ of the process parameter. But in this case, an operational margin $\Delta_{y H}$ for deviation of the parameter of technological process from the adjusting value $y_{H}$ is created. Value of controlled level ${ }_{\Delta H}$ of preservation of operational margin and its components is determined by the following expressions:

$$
\left.\begin{array}{c}
P_{\Delta \mathrm{H}}=\int_{y_{i}-\Delta_{j i}}^{y_{i}+\Delta_{y i}} f(y) d y=F\left(y_{i}+\Delta_{y i}\right)-F\left(y_{i}-\Delta_{y i}\right) \\
P_{\Delta \mathrm{H}}^{-}=\int_{y_{i}-\Delta_{y i}}^{y_{y}} f(y) d y=F\left(y_{i}\right)-F\left(y_{i}-\Delta_{y i}\right) \\
P_{\Delta \mathrm{H}}^{+}=\int_{y_{i}}^{y_{i}+\Delta_{y i j}} f(y) d y=F\left(y_{i}+\Delta_{y i}\right)-F\left(y_{i}\right)
\end{array}\right\}
$$

where:

$F(z)$ - one-dimensional distribution function of the corresponding argument $z$.

Due to shift of adjusting value $y_{H}$ from average value ${ }^{m_{y}}$ of realization $y(t)$, a mismatch appears

$$
{ }_{\Delta} P=P_{\Delta H}^{-}+P_{\Delta H}^{+}=2 F\left(y_{H}\right)-\left[F\left(y_{H}+\Delta_{y H}\right)+F\left(y_{H}-\Delta_{y H}\right)\right] .
$$

With normal arrangement of expression (7) takes the following form:

$$
\begin{aligned}
& P_{\Delta \mathrm{H}}^{-}=\Phi\left(\frac{\Delta_{i}}{\sigma_{y}}\right)+\Phi\left[\frac{\left(\Delta_{y i}-\Delta_{i}\right)}{\sigma_{y}}\right] \\
& \left.P_{\Delta \mathrm{H}}^{+}=\Phi\left[\frac{\left(\Delta_{y i}+\Delta_{i}\right)}{\sigma_{y}}\right]-\Phi\left(\frac{\Delta_{i}}{\sigma_{y}}\right)\right\}, \\
& { }_{\Delta} P=P_{\Delta \mathrm{H}}^{-}-P_{\Delta \mathrm{H}}^{+}=2 \Phi\left(\frac{\Delta_{y i}}{\sigma_{y}}\right)-{ }_{\Delta} E,
\end{aligned}
$$

Expression for calculating the estimated probability of overshoot $\varepsilon_{\Delta \mathrm{H}}^{-} \grave{e} \varepsilon_{\Delta \mathrm{H}}^{+}$with normal layout has the following form:

$$
\left.\begin{array}{l}
\mathcal{E}_{\Delta \mathrm{H}}^{-}=0.5-\Phi\left[\frac{\Delta_{y i}-\Delta_{i}}{\sigma_{y}}\right] \\
\varepsilon_{\Delta \mathrm{H}}^{+}=0.5+\Phi\left[\frac{\Delta_{y i}+\Delta_{i}}{\sigma_{y}}\right]
\end{array}\right\},
$$

General estimated probability of overshoot above level $y_{H}$ will be

$$
\varepsilon_{\Delta \mathrm{H}}=\varepsilon_{\Delta \mathrm{H}}^{-}+\varepsilon_{\Delta \mathrm{H}}^{+}=1-P_{\Delta \mathrm{H}} \text {. }
$$

Estimation of overshoot ${ }^{n \Delta_{i}}$ above the absolute margin with normal layout may be found with the help of expression $n_{\Delta}=\frac{\omega_{k}}{2 \pi} \exp \left[-\frac{\left(\Delta_{i} \pm \Delta_{o i}\right)^{2}}{2 \sigma_{o}^{2}}\right]$ 


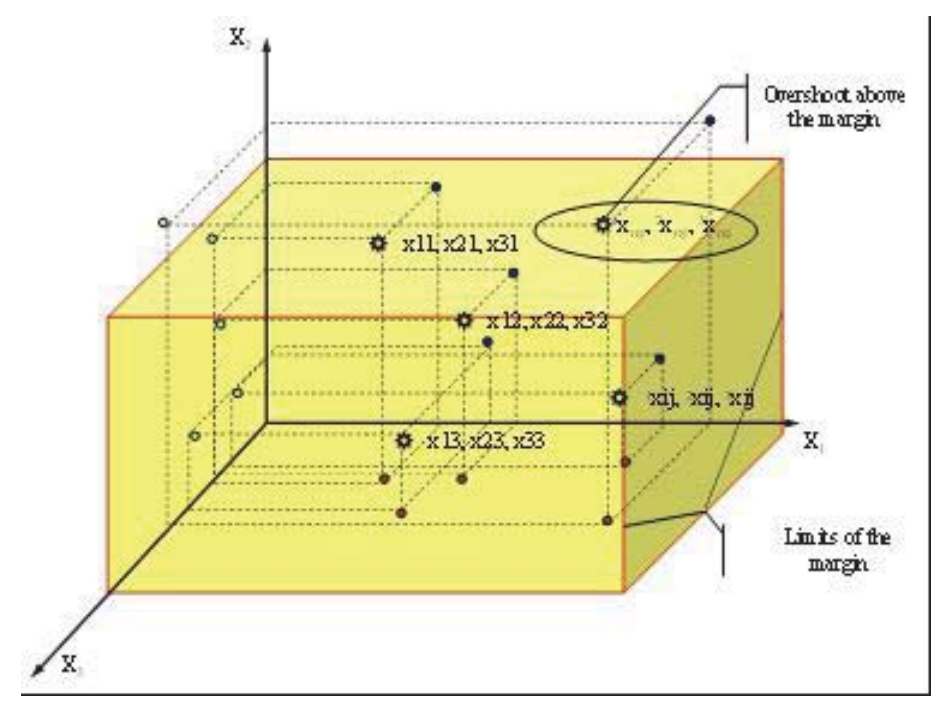

Fig. 3. Quantity of overshoots ${ }^{n \Delta_{i}}$ above the absolute margin with normal layout

Correlation of (10) and (11) may be the basis of not only the algorithm of operating control of relative duration $P_{\Delta H}$ of the parameter of cattle-breeding enterprise technological process in the margin zone, but of the algorithm of management of this process's quality, as the signal ${ }_{\Delta} P\left({ }_{0}{ }^{\varepsilon} \varepsilon\right)$ determines the operator and relative magnitude of deviation of actual value $P_{\Delta H}$ from the set $P_{\Delta 3}$. Thus, a task of the management system which realizes this algorithm is not the decrease of deviations of initial parameter $y(t)$ from its adjusting value $y_{r}$, but preservation of the highest value ${ }_{\Delta H}$ (or the lowest value) $\varepsilon_{\Delta \mathrm{H}}$, which takes place with ${ }_{\Delta} P \rightarrow 0$.

This method is used for forecasting and controlling the process of observation at the researched TEES.

Every stage of the management of enterprise (sector) features uncertainty and mistakes which are caused by the relativity of knowledge of the management subject (supervising manpower) of the condition of the object under management, of condition of external environment and its influence on the object under management. That's why the probabilistic nature of achieving the goal, determined by the described above uncertainty in the goal setting and its probable perturbation, may be characterized by the notion of risk.

In order to provide stable development of enterprise, it is important not only to quickly identify and effectively solve all the problems, but also foresee and prevent their appearance and take into account the level of influence on the technical and economic parameters. It is also very important to have reserve for financing the risks that cannot be prevented. Especially it relates the issues which may threaten the very existence of the enterprise. Creating a system for risks management is a logical stage of development of modern enterprise management system. Risk management becomes more actual after detection of the risk-problem $[3,4]$. Risk may be described by two probabilities - probability $p 1$ of appearance of unfavorable process as such and probability $p 2$ of this process making maximal damage $U$ to the object of management at set confidence level. The goal of risk management is to influence the external and internal environment of the organization in such a way, so that the possible damage would be of acceptable level.

For estimating the risks $R$, the functional $F$ is used which connects the probability $P$ of the process overshoot above margin and mathematical expectation of damage $U$ from this unfavorable event

$$
R=F_{R}\{U, P\}=\sum_{i}\left[F_{R_{i}}\left(U_{i}, P_{i}\right)\right]=\int C(U) P(U) d U=\int C(P) U(P) d P \text {, }
$$

where:

$i$ - types of unfavorable events;

$\mathrm{C}$ - weight functions, taking into account the mutual influence of risks.

Generally, for qualitative and quantitative analysis of risks according to expression (13) on the basis of studying complex dynamic processes (appearance of violations, i.e. overshoot above margink), physical and mathematical models are built. In this approach, time scales of risks $R(t)$ are used. 
Total damage $U$ (or its components $U_{i}$ ) is found through extended functional (sum) of damages, sustained by technical and eco-economical system of enterprise:

$$
U=F_{U}\left\{U_{T}, U_{\hat{\jmath}}, U_{\ni}\right\}=\sum_{i}\left[F_{U_{i}}\left(U_{T_{i}}, U_{\hat{\vartheta}_{i}}, U_{\vartheta_{i}}\right)\right] \text {. }
$$

Damages $U$ according to (14) and, respectively, risks $R$ according to (13) are found with the large amount of factors.

Probability $P$ of appearance of unfavorable event (or its components $P_{i}$ ) is usually defined as a functional of probabilities, depending on the sources of corresponding factors: technological $\rightarrow T$, ecological $\rightarrow \Im^{*}$ and economic $\rightarrow \ni$.

$$
P=F_{p}\left\{P_{T}, P_{\Im}, P_{\ni}\right\}=\sum_{i}\left[F_{P_{i}}\left(P_{T_{i}}, P_{丂_{i}}, P_{\ni_{i}}\right)\right] .
$$

At that, the sufficient conditions of structural dynamic and statistic sustainability may be determined at any stage of structural transformations.

Algorithm for estimation of structural dynamic and statistic sustainability of enterprise (sector) is shown in Fig. 4.

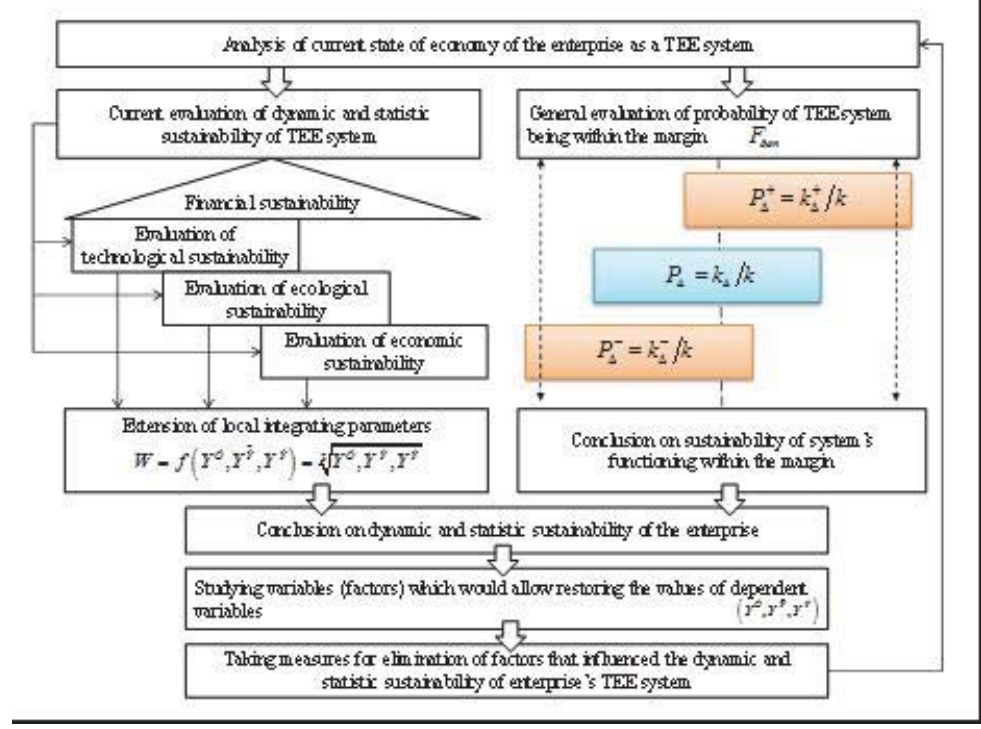

Fig. 4. Algorithm of evaluation of structural dynamic and statistic sustainability of the enterprise (sector)

\section{Conclusion}

After quantitative determination of general evaluation of probability of TEES being within the allowed limits, the values of risks of various variants of solution are compared, and the one that corresponds to the chosen risk strategy of the enterprise is chosen.

The sense of the methods consists in the fact that for each situation a computer determines the level of its probability (e.g., low probability, average probability, high probability) and potential damage which corresponds to that situation (e.g., small, medium, large).

\section{References}

Kudryavskiy Y.P., Shchetkin B.N. et al. (1995). Methods for preparing bird droppings for utilization. Patent RF №2090540, appl. №95117662, 1995.

Lurye A.B. (1981). Statistical dynamics of agricultural machines. - M.: Kolos, 1981. -382 p.

Shchetkin B.N. (2002). Utilization of poultry industry waste - solution to the problems of ecological security and resource economy. Perm: OGUP "Solikamsk printing office", 2002. - 135 p.

Yandyganov Y.Y., Kozitsin A.A., Nosov A.A., Fedorov M.V. (2002). Ecological risks (evaluation and insurance mechanism). Ekaterinburg. Ural State Economic University publishing house. 2002. - 222 p. 